\title{
Inversion of the Circular Averages Transform using the Funk Transform
}

\author{
Can Evren Yarmanłand Birsen Yazıcı $\Varangle$, \\ $\dagger$ Westerngreco - Schlumberger, Houston, TX 77042 \\ $\ddagger$ Department of Electrical, Computer and System Engineering, Rensselaer Polytechnic \\ Institute, Troy, NY 12180-3590, USA
}

\begin{abstract}
.
The integral of a function defined on the half-plane along the semi-circles centered on the boundary of the half-plane is known as the circular averages transform. Circular averages transform arises in many tomographic image reconstruction problems. In particular, in Synthetic Aperture Radar (SAR) when the transmitting and receiving antennas are colocated, the received signal is modeled as the integral of the ground reflectivity function of the illuminated scene over the intersection of spheres centered at the antenna location and the surface topography. When the surface topography is flat the received signal becomes the circular averages transform of the ground reflectivity function. Thus, SAR image formation requires inversion of the circular averages transform. Apart from SAR, circular averages transform also arises in thermo-acoustic tomography and sonar inverse problems.

In this paper, we present a new inversion method for the circular averages transform using the Funk transform. For a function defined on the unit sphere, its Funk transform is given by the integrals of the function along the great circles. We used hyperbolic geometry to establish a diffeomorphism between the circular averages transform, hyperbolic $\mathrm{x}$-ray and Funk transforms. The method is exact and numerically efficient when fast Fourier transforms over the sphere are used. We present numerical simulations to demonstrate the performance of the inversion method.
\end{abstract}

$\S$ To whom correspondence should be addressed (yazici@ecse.rpi.edu) 
This work is dedicated to Dennis Healy, a friend of applied mathematics \& engineering.

\section{Introduction}

The circular averages transform arises in many inverse problems including Synthetic Aperture Radar (SAR) [1, 2], thermo-acoustic tomography [3] and sonar [4].

In SAR, when the wavelength is considerably longer than the size of the antenna, the transmitted electromagnetic waves are almost spherical. For an aircraft cruising along a straight flight trajectory at a constant altitude with constant velocity, the received signal from a flat topography can be modeled by the circular averages transform of the ground reflectivity function $[5,1]$. Here, the center of the circles corresponds to the projection of the flight path onto the ground as illustrated in Figure 1.

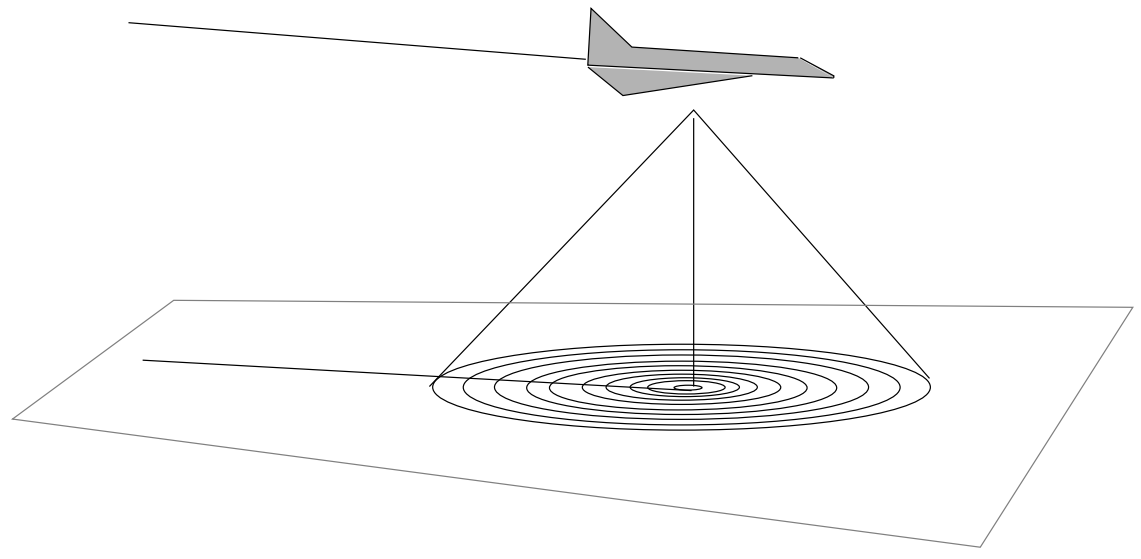

Figure 1. An illustration of the synthetic aperture radar geometry.

Without loss of generality, assuming that the flight trajectory is along $x_{1}$-axis, the circular averages transform of the ground reflectivity $f$ is given by

$$
g(c, t)=\mathcal{R}_{C}[f](c, t)=\int_{0}^{2 \pi} f(c+t \cos \theta, t \sin \theta) d \theta
$$

where $c \in \mathbb{R}$ and $t \in \mathbb{R}^{+}$denote the center on $x_{1}$-axis and the radius of the circle, respectively. Thus, the reconstruction of the ground reflectivity function from SAR data requires inversion of the circular averages transform.

If a function $f$ is odd in its second variable, i.e., $f\left(x_{1}, x_{2}\right)=-f\left(x_{1},-x_{2}\right)$, then its circular averages transform vanishes. The unique determination of a function $f$, which is symmetric with respect to $x_{1}$-axis, from its circular averages transform $g$ was proved by Courant and Hilbert [6]. As a consequence, for a side looking radar, a function supported on the half-plane can be uniquely recovered from its integrals over the semi-circles centered on the boundary of the half-plane. For the rest of our discussion, we consider inversion of $\mathcal{R}_{C}$ for functions that are symmetric with respect to $x_{1}$-axis. In this regard, we do not make any distinction between the circular averages transform of even functions and semi-circular averages transform of functions supported on the upper half-plane. 
In [7], Lavrent'ev gave a characterization of the range of the circular averages transform and an inversion method that requires infinite number of the moments of $\mathcal{R}_{C}[f]$. One of the earliest inversion methods for circular averages transform was presented by Norton where weighted parameterized projections were related to the weighted parameterized function via a two dimensional convolution (see equation (16) in [8]) and the inversion was derived using the 2D Fourier transform. Another inversion method based on the 2D Fourier transform was given by Palamodov [9]. An alternative inversion was presented by Fawcett where he showed that the Fourier-Hankel transform of $\mathcal{R}_{C}[f]$ is equivalent to the two-dimensional Fourier transform of $f$ (see equation (11a) in [10]). Andersson gave an alternative proof of Fawcett's inversion method, by taking the Fourier transform of $\mathcal{R}_{C}[f]$ [1]. The first fast inversion algorithm for the circular averages transform was developed by Nilsson, who reformulated Andersson's inversion formula in a filtered-backprojection form [11]. For a more detailed review of the circular averages transform inversion methods, see [12].

In this paper, we develop a new inversion method for the circular averages transform using the Funk transform, where we use results from the hyperbolic geometry to express the circular averages transform in terms of the Funk transform. For a function defined on the unit sphere, its Funk transform is given by the integrals of the function along the great circles. Expressing the circular averages transform in terms of the hyperbolic x-ray transform on the hyperbolic half-plane, we derive a diffeomorphism that transforms the hyperbolic $\mathrm{x}$ ray transform on the hyperbolic half-plane into the Funk transform. Thus, we show that the inversion of the circular averages transform is equivalent to the inversion of the Funk transform and use fast Fourier transforms over the rotation group to invert the circular averages transform. We present numerical simulations to demonstrate the applicability of the new inversion method.

The organization of the paper is as follows: In Section 2, we present the Funk transform and its inversion. In Section 3, we present the relationship between the upper semi-sphere and hyperbolic half-plane. In Section 4, we give an inversion algorithm for the circular averages transform using the results in Section 3. In Section 5, we demonstrate the performance of the inversion method in numerical simulations. Finally, we conclude our discussion in Section 6. The paper includes an appendix presenting an intermediate result needed for the inversion method.

\section{The Funk Transform and its Inversion}

We use the following notational conventions throughout the paper. The bold letters, such as $\boldsymbol{x}, \boldsymbol{n}$ etc. are used to denote vector quantities. The calligraphic letters $(\mathcal{F}, \mathcal{K}$ etc. $)$ are used to denote operators. Table 1 lists the notations used throughout the paper.

Given a function $f$ defined on the unit sphere $S^{2}=\left\{\boldsymbol{x} \in \mathbb{R}^{3}:|\boldsymbol{x}|=1\right\}$, its Funk transform is defined by the integrals of $f$ along the great circles:

$$
\mathcal{M}[f](\boldsymbol{n})=\int_{S^{2}} f(\boldsymbol{x}) \delta(\boldsymbol{x} \cdot \boldsymbol{n}) d \boldsymbol{x}
$$

where $\delta$ is the one dimensional Dirac-delta function, $d \boldsymbol{x}$ is the normalized Haar measure on 
Table 1. Table of Notations

\begin{tabular}{|c|c|}
\hline Symbol & Designation \\
\hline $\mathcal{M}$ & Funk transform \\
\hline$S^{2}$ & Unit sphere \\
\hline$\delta$ & One-dimensional Dirac-delta function \\
\hline$n$ & Normal of the plane enclosing a great circle \\
\hline$\theta$ & An element of $S^{2}$ \\
\hline$S O(3)$ & Three-dimensional rotation group \\
\hline$e_{i}$ & $i^{t h}$ column of the 3 -by-3 identity matrix \\
\hline$d(Z)$ & Normalized Haar measure on $\mathrm{SO}(3)$ \\
\hline$\alpha, \gamma, \beta$ & Euler angles in $[0,2 \pi)$ and $[0, \pi)$ \\
\hline$\hat{\Lambda}$ & $\begin{array}{l}S O(3) \text {-Fourier transform of a function } \Lambda \text { defined on } \\
S O(3)\end{array}$ \\
\hline$\hat{\Lambda}_{k l}$ & Matrix coefficients of $\hat{\Lambda}$ \\
\hline$S_{+}^{2}$ & Unit upper hemisphere \\
\hline$H_{+}^{2}$ & $\begin{array}{l}\text { The upper sheet of the two-sheet hyperboloid, }\left\{\boldsymbol{x} \in \mathbb{R}^{3} \text { : }\right. \\
\left.x_{1}^{2}+x_{2}^{2}-x_{3}^{2}=-1\right\}\end{array}$ \\
\hline$C_{+}$ & The cone $\left\{\boldsymbol{x} \in \mathbb{R}^{3}: x_{3}>0, \arccos \left(x_{3} /|\boldsymbol{x}|\right)<\pi / 4\right\}$ \\
\hline$\pi_{S_{+}^{2}}$ & $\begin{array}{l}\text { Projection operator from the upper-half space, } \\
\left\{\left(x_{1}, x_{2}, x_{3}\right): x_{3}>0\right\} \text {, onto the upper hemisphere } \\
S_{+}^{2}\end{array}$ \\
\hline$\pi_{H_{+}^{2}}$ & $\begin{array}{l}\text { Projection operator from the cone } C_{+} \text {onto the hyper- } \\
\text { boloid } H_{+}^{2}\end{array}$ \\
\hline$\pi_{(-1,0,0)}$ & Projection onto Hyperbolic Half-plane \\
\hline$\pi_{\left(H_{+}^{2} \rightarrow S_{+}^{2}\right)}$ & Projection operator from $H_{+}^{2} \rightarrow S_{+}^{2}$ \\
\hline$H_{+}$ & The half plane defined by $\left\{\left(x_{1}, x_{2}, x_{3}\right): x_{1}=1, x_{3}>0\right\}$ \\
\hline$\Phi$ & $\pi_{(-1,0,0)} \circ \pi_{\left(H_{+}^{2} \rightarrow S_{+}^{2}\right)} \circ \pi_{H_{+}^{2}}$ \\
\hline$\Phi^{*}$ & Pull-back of $\Phi$ \\
\hline$\|x\|_{H}$ & Hyperbolic metric, $\sqrt{x_{1}^{2}+x_{2}^{2}-x_{3}^{2}}$ \\
\hline$d s_{H_{+}}^{2}$ & Riemannian metric on $H_{+}$ \\
\hline$d s_{C_{+} \cap S_{+}^{2}}^{2}$ & Riemannian metric on $C_{+} \cap S_{+}^{2}$ \\
\hline
\end{tabular}

$S^{2}[13]$ and $\boldsymbol{n}$ is the normal of the plane enclosing a great circle as illustrated in Figure 2.

By (2), the Funk transform is even, i.e., $\mathcal{M}[f](-\boldsymbol{n})=\mathcal{M}[f](\boldsymbol{n})$. Therefore, only the even part of a function can be uniquely recovered from its Funk transform. In [14], Funk showed that the even part of a function over $S^{2}$ can be uniquely determined from its Funk transform and presented two different inversion methods. The first method is based on expressing the spherical harmonic decomposition of the function in terms of the spherical harmonic decomposition of its Funk transform. The second method utilizes Abel's integral equation. We focus on the first inversion method since it can be efficiently implemented using the fast Fourier transform algorithms over the rotation group and the sphere $[15,16,17,18,19,20],[21,22]$. 


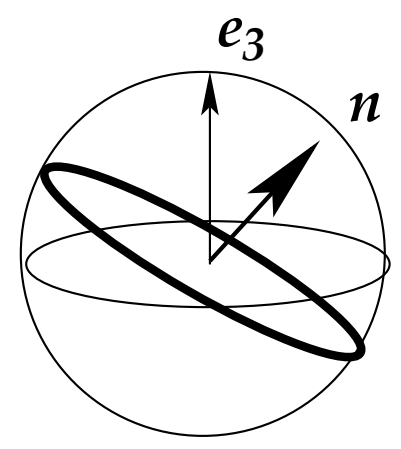

Figure 2. The geometry of the Funk transform. The vector $\boldsymbol{n}$, the normal of the plane enclosing a great circle, parametrizes the Funk transform.

By (2), the Funk transform is invariant with respect to rotations, i.e., $\mathcal{M}[f(Q \cdot)](\boldsymbol{\theta})=$ $\mathcal{M}[f](Q \boldsymbol{\theta})$, where $\boldsymbol{\theta} \in S^{2}$ and $Q \in S O(3)$ is a rotation matrix $\dagger$.

Let $\boldsymbol{e}_{\boldsymbol{i}}, i=1,2,3$, denote the $i^{\text {th }}$ column of the 3 -by-3 identity matrix. Since $S^{2}$ is a homogeneous space of $S O(3)$, any unit vector can be written as a product of a rotation matrix and the unit vector $e_{3}$. Thus, using the homogeneity of $S^{2}$, we can express the Funk transform as a convolution over the rotation group $S O(3)$ as follows:

$$
\begin{aligned}
\mathcal{M}[f](\boldsymbol{n}) & =\int_{S^{2}} f(\boldsymbol{x}) \delta\left(\boldsymbol{x} \cdot \boldsymbol{R e}_{\mathbf{3}}\right) d \boldsymbol{x} \\
& =\int_{S O(3)} F(Z) \Lambda_{\mathcal{M}}\left(R^{-1} Z\right) d(Z) \\
& =\left(F *_{S O(3)} \Lambda_{\mathcal{M}}^{*}\right)(R),
\end{aligned}
$$

where $R, Z \in S O(3), \boldsymbol{n}=\operatorname{Re}_{\mathbf{3}}, F(Z)=f\left(Z \boldsymbol{e}_{\mathbf{3}}\right), \delta\left(\boldsymbol{s} \cdot \boldsymbol{e}_{\mathbf{3}}\right)$ is the Dirac measure of the equatorial circle, $\Lambda_{\mathcal{M}}(Z)=\delta\left(Z \boldsymbol{e}_{3} \cdot \boldsymbol{e}_{3}\right)$ is the kernel of the Funk transform, and $d(Z)$ is the normalized Haar measure on $S O(3)$. Using the Euler angles $\alpha, \gamma \in[0,2 \pi)$, and $\beta \in[0, \pi)$, the normalized Haar measure on $S O(3)$ can be expressed as $d \alpha \sin \beta d \beta d \gamma$.

Let $\widehat{\Lambda}$ denote the $S O(3)$-Fourier transform of the function $\Lambda$ defined on $S O(3)$ and $\widehat{\Lambda}_{k l}$ represent the matrix coefficients of $\widehat{\Lambda}$. By the $S^{2}$-invariance and the convolution property of the $S O(3)$-Fourier transform [23], the Funk transform is diagonalized in the $S O(3)$-Fourier domain as follows:

$$
{\widehat{\mathcal{M}}[f]_{m 0}^{2 l}}^{2 l}=\widehat{F}_{m 0}^{2 l}{\overline{\widehat{\Lambda}_{\mathcal{M}} 00}}^{2 l},
$$

where

$$
\begin{aligned}
\widehat{F}_{m n}^{l}=\frac{2 l+1}{8 \pi^{2}} \int_{0}^{2 \pi} \int_{0}^{\pi} & \int_{0}^{2 \pi} F(\alpha, \beta, \gamma) \mathrm{e}^{\mathrm{i}(m \alpha+n \gamma)} \\
& \times \overline{d_{m n}^{l}(\beta)} d \alpha \sin \beta d \beta d \gamma, \quad|m|,|n| \leq l \in \mathbb{Z}^{+},
\end{aligned}
$$

$\dagger S O(3)$ is a three-parameter Lie group whose elements are 3-by-3 real orthogonal matrices with determinant equal to 1 . 
and

$$
F(\alpha, \beta, \gamma)=\sum_{l \geq 0} \sum_{m=-l}^{l} \sum_{n=-l}^{l} \widehat{F}_{m n}^{l} \mathrm{e}^{-\mathrm{i}(m \alpha+n \gamma)} d_{m n}^{l}(\beta)
$$

are the matrix coefficients of the $S O(3)$-Fourier and inverse $S O(3)$-Fourier transforms of $F$, respectively. Here, $d_{m n}^{l}$ are the Wigner-D functions $[24,25]$. Thus, for an even function $f$ on the unit sphere, the inversion of the Funk transform can be implemented efficiently using the fast $S O(3)$-Fourier transform algorithms [19, 20, 21, 22] in three steps as shown below:

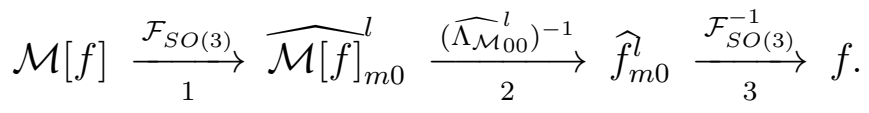

Due to the evenness of the Funk transform of a function, for the rest of our paper, we consider only the functions defined on the upper-hemisphere of $S^{2}$ which can be uniquely recovered from their Funk transforms.

\section{Mapping from the Upper Semi-Sphere to the Hyperbolic Half-Plane}

In this section, we present some results from the hyperbolic geometry [26, 27, 28] and use these results to establish a relationship between the Funk and circular averages transforms.

\subsection{Mapping from the Upper-Hemisphere to the Upper-Hyperboloid}

Let $\boldsymbol{x} \in \mathbb{R}^{3}, x_{i}=\boldsymbol{x} \cdot \boldsymbol{e}_{\boldsymbol{i}}, i=1,2,3$; and $S_{+}^{2}=\left\{\boldsymbol{x} \in S^{2}: x_{3}>0\right\}$ be the unit upperhemisphere. We define $\pi_{S_{+}^{2}}:\left\{\boldsymbol{x} \in \mathbb{R}^{3}: x_{3}>0\right\} \rightarrow S_{+}^{2}$ to be the mapping

$$
\pi_{S_{+}^{2}}(\boldsymbol{x})=\frac{\boldsymbol{x}}{|\boldsymbol{x}|}
$$

that projects a point in the upper-half space $x_{3}>0$ onto the upper-hemisphere $S_{+}^{2}$ (see Figure $11)$.

Let $H_{+}^{2}=\left\{\boldsymbol{x} \in \mathbb{R}^{3}: x_{1}^{2}+x_{2}^{2}-x_{3}^{2}=-1, \quad x_{3}>0\right\}$ be the upper-sheet of the two-sheet hyperboloid, as shown in Figure 11. Let $C_{+}$denote the cone defined by $C_{+}=\left\{\boldsymbol{x} \in \mathbb{R}^{3}: x_{3}>0, \arccos \left(x_{3} /|\boldsymbol{x}|\right)<\pi / 4\right\}$. We define $\pi_{H_{+}^{2}}: C_{+} \rightarrow H_{+}^{2}$ to be the mapping

$$
\pi_{H_{+}^{2}}(\boldsymbol{x})=\frac{\boldsymbol{x}}{\sqrt{x_{3}^{2}-x_{1}^{2}-x_{2}^{2}}}, \quad x_{i}=\boldsymbol{x} \cdot \boldsymbol{e}_{\boldsymbol{i}}, \quad i=1,2,3,
$$

that projects any point $\boldsymbol{x} \in C_{+}$onto the hyperboloid $H_{+}^{2}$. Thus, $\pi_{H_{+}^{2}}$ projects the arcs of the semi-great circles of $S_{+}^{2}$ lying in $C_{+}$onto the hyperbolas that are given by the intersection of the hyperboloid $H_{+}^{2}$ with the plane containing the semi-great circles. These hyperbolas are called the geodesic lines of the hyperbolic plane [27] (see Figure 11).

\subsection{Mapping from the Upper-Hyperboloid to the Upper Semi-Sphere via Poincaré Disk}

Consider the line segment that connects the points $\boldsymbol{x}=\left(x_{1}, x_{2}, x_{3}\right) \in H_{+}^{2}$ and $\left(x_{1}, x_{2}, 0\right) /(1+$ $x_{3}$ ), which lies within the unit disk on the plane $x_{3}=0$. The intersection of this line segment 


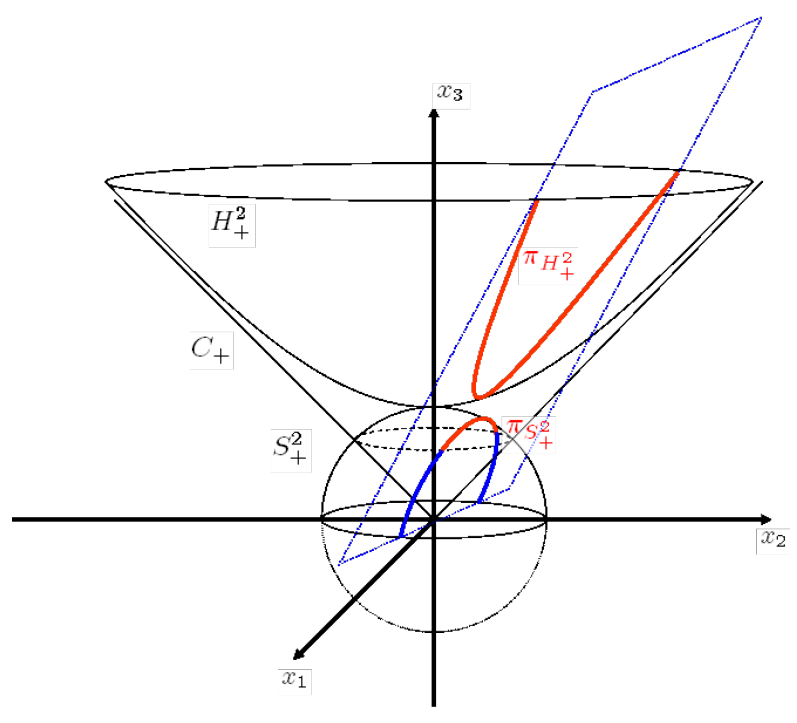

Figure 3. $\pi_{H_{+}^{2}}$ projects arcs of semi-great circles of $S_{+}^{2}$ lying in $C_{+}$onto the hyperbolas given by the intersection of the plane containing the semi-great circle and $H_{+}^{2}$. Conversely, $\pi_{S_{+}^{2}}$ projects hyperbolas obtained by the intersection of $H_{+}^{2}$ and planes passing from the origin onto the arcs of semi-great circles that lie in these planes and $C_{+}$.

with $S_{+}^{2}$ is given by $\left(x_{1}, x_{2}, 1\right) / x_{3}$. As a consequence, the mapping $\pi_{\left(H_{+}^{2} \rightarrow S_{+}^{2}\right)}: H_{+}^{2} \rightarrow S_{+}^{2}$ defined by

$$
\pi_{\left(H_{+}^{2} \rightarrow S_{+}^{2}\right)}(\boldsymbol{x})=\left(x_{1} / x_{3}, x_{2} / x_{3}, 1 / x_{3}\right), \quad x_{i}=\boldsymbol{x} \cdot \boldsymbol{e}_{\boldsymbol{i}}, \quad i=1,2,3
$$

maps the geodesic lines of $H_{+}^{2}$ to the semi-circles which are given by the intersection of $S_{+}^{2}$ and the planes perpendicular to $x_{1}-x_{2}$ plane [27] (see Figure 4).

Let $H_{+}$denote the half-plane $x_{1}=1, x_{3}>0$. The projection of the resulting semi-circles onto $H_{+}$using the mapping $\pi_{(-1,0,0)}: S_{+}^{2} \rightarrow H_{+}$defined by

$$
\pi_{(-1,0,0)}(\boldsymbol{x})=\left(1, \frac{2 x_{2}}{1+x_{1}}, \frac{2 x_{3}}{1+x_{1}}\right), \quad x_{i}=\boldsymbol{x} \cdot \boldsymbol{e}_{\boldsymbol{i}},
$$

gives semi-circles that have their centers on (or half-lines that are perpendicular to) the line $x_{1}=1, x_{2} \in \mathbb{R}, x_{3}=0$ [27]. Thus, $\pi_{(-1,0,0)} \circ \pi_{\left(H_{+}^{2} \rightarrow S_{+}^{2}\right)}$ maps $H_{+}^{2}$ onto the plane $x_{1}=1$ (see Figure 5). Note that the half-lines are semi-circles with infinite radius. In the context of SAR, we are not interested in the half-lines but only the semi-circles since we can only collect data for a finite time duration, which is related to the maximum radius of the semi-circles that are within the scene to be imaged (see (1)).

\subsection{Mapping from the Upper Semi-Sphere to the Hyperbolic Upper Half-plane}

Let $\Phi: S_{+}^{2} \cap C_{+} \rightarrow H_{+}$be defined by

$$
\Phi(\boldsymbol{x})=\pi_{(-1,0,0)} \circ \pi_{\left(H_{+}^{2} \rightarrow S_{+}^{2}\right)} \circ \pi_{H_{+}^{2}}(\boldsymbol{x}),
$$




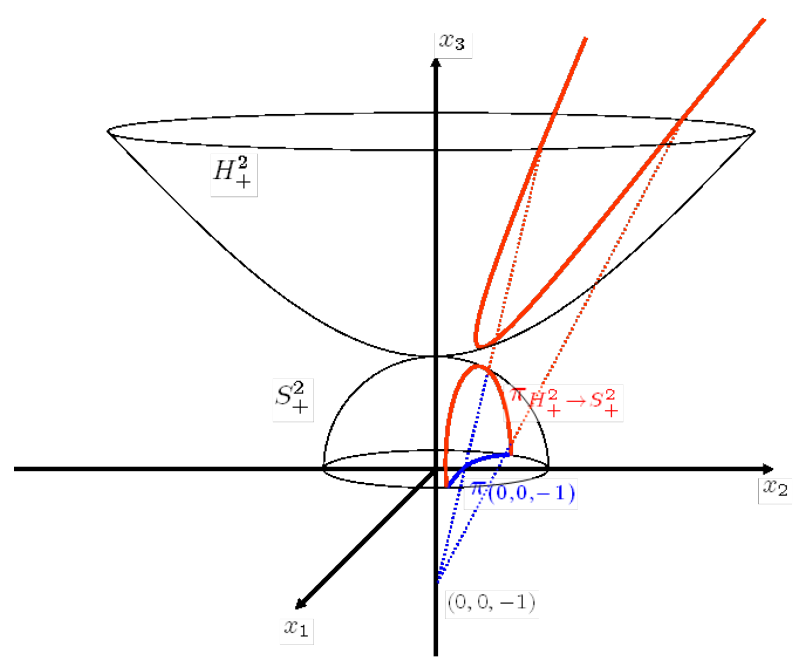

Figure 4. The projection of $H_{+}^{2}$ onto the upper-hemisphere by $\pi_{H_{+}^{2} \rightarrow S_{+}^{2}}$. The geodesic hyperbolas on $H_{+}^{2}$ are mapped onto the semi-circles obtained by the intersection of the plane perpendicular to the $x_{1}-x_{2}$ plane with $S_{+}^{2}$.
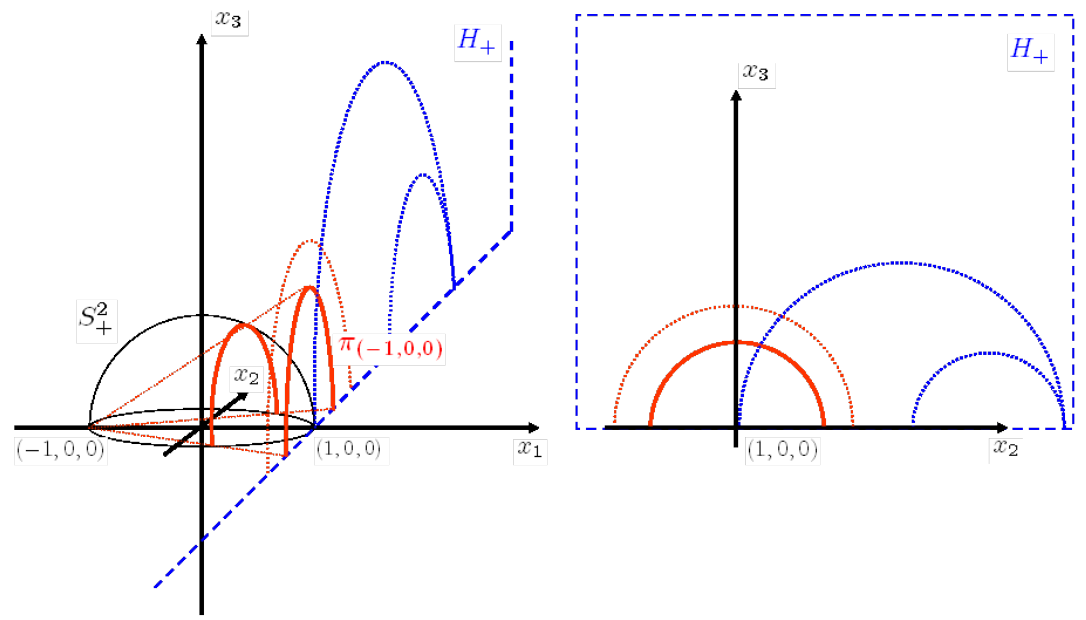

Figure 5. The projection of the upper-hemisphere onto the hyperbolic half-plane $H_{+}$ by $\pi_{(-1,0,0)} \cdot \pi_{(-1,0,0)}$ maps the semi-circles obtained by the intersection of the plane perpendicular to the $x_{1}-x_{2}$ plane with $S_{+}^{2}$ onto the semi-circles centered on the line $x_{1}=1$, $x_{2} \in \mathbb{R}, x_{3}=0$. 
Inversion of the Circular Averages Transform using the Funk Transform

$$
=\left(1, \frac{2 x_{2}}{x_{1}+x_{3}}, \frac{2 \sqrt{x_{3}^{2}-\left(x_{1}^{2}+x_{2}^{2}\right)}}{x_{1}+x_{3}}\right)=\left(1, y_{2}, y_{3}\right) \text {. }
$$

Since $\pi_{H_{+}^{2}}$ projects the arcs of the semi-great circles at the intersection of $S_{+}^{2}$ and the cone $C_{+}$onto the geodesics of the hyperboloid $H_{+}^{2}, \Phi$, defined by the composition of $\pi_{H_{+}^{2}}$ with $\pi_{(-1,0,0)} \circ \pi_{\left(H_{+}^{2} \rightarrow S_{+}^{2}\right)}$, maps $C_{+} \cap S_{+}^{2}$ diffeomorphically onto $H_{+}$, while mapping the arcs of the semi-great circles of $S_{+}^{2}$ lying in $C_{+}$to the semi-circles centered on the boundary of $H_{+}$ as illustrated in Figure 6 . Note that we drop 1 and identify $\left(1, y_{2}, y_{3}\right)$ with $\boldsymbol{y}=\left(y_{2}, y_{3}\right)$, and treat $\boldsymbol{y}$ as a point on $H_{+}$.

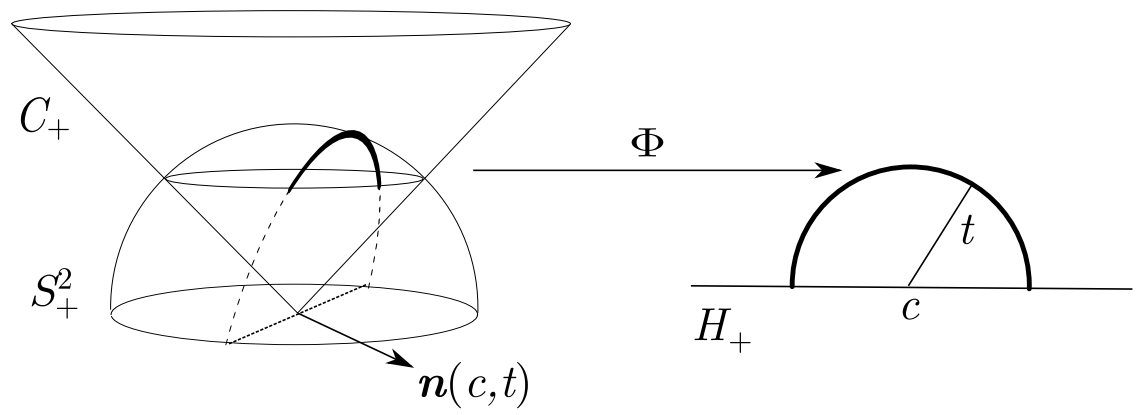

Figure 6. An illustration of the mapping $\Phi$ from $C_{+} \cap S_{+}^{2}$ to $H_{+}$.

Conversely, both $\pi_{(-1,0,0)}$ and $\pi_{\left(H_{+}^{2} \rightarrow S_{+}^{2}\right)}$ are invertible. Therefore, $\pi_{\left(H_{+}^{2} \rightarrow S_{+}^{2}\right)}^{-1} \circ \pi_{(-1,0,0)}^{-1}$ maps the semi-circles centered on the boundary of $H_{+}$onto the hyperbolas formed by the intersection of the upper-sheet $H_{+}^{2}$ and the plane passing through the origin with the normal $\boldsymbol{n}$ (see Figure 7). As a consequence, $\Phi^{-1}=\pi_{S_{+}^{2}} \circ \pi_{\left(H_{+}^{2} \rightarrow S_{+}^{2}\right)}^{-1} \circ \pi_{(-1,0,0)}^{-1}$ maps the semi-circles centered on the boundary of $H_{+}$onto the arcs of the semi-great circles of $S_{+}^{2}$ lying in $C_{+}$(see Figure 7).

\section{Inversion of the Circular Averages Transform}

\subsection{Mapping from the Funk Transform to the Circular Averages Transform}

Integration of a function $f$ along the semi-circles centered on the boundary of $H_{+}$is known as the X-ray transform on the hyperbolic half-plane or hyperbolic x-ray transform on the upper half-plane [29]. Since the projection $f(\Phi(\boldsymbol{x}))$ of a function $f$ on $H_{+}$back onto $C_{+} \cap S_{+}^{2}$ can be treated as a function on $S^{2}$ supported on $C_{+} \cap S_{+}^{2}$, taking the hyperbolic x-ray transform of $f$ on $H_{+}$, is equivalent to taking the weighted Funk transform of the compactly supported function $f(\Phi(\boldsymbol{x})), \boldsymbol{x} \in C_{+} \cap S_{+}^{2}$, on $S^{2}$ :

Lemma 4.1 Given a great circle $Y$ perpendicular to the unit vector $\boldsymbol{n}$, we have

$$
\begin{aligned}
\int_{\Phi\left(Y \cap C_{+} \cap S_{+}^{2}\right)} f\left(y_{2}, y_{3}\right) \sqrt{d s_{H_{+}}^{2}} & =\int_{Y \cap C_{+} \cap S_{+}^{2}} \Phi^{*} f\left(y_{2}, y_{3}\right) \sqrt{\Phi^{*} d s_{H_{+}}^{2}} \\
& =\int_{Y \cap C_{+} \cap S_{+}^{2}} f(\Phi(\boldsymbol{x})) \frac{\|\boldsymbol{n}\|_{H}}{\|\boldsymbol{x}\|_{H}^{2}} \sqrt{d s_{C_{+} \cap S_{+}^{2}}^{2}}
\end{aligned}
$$




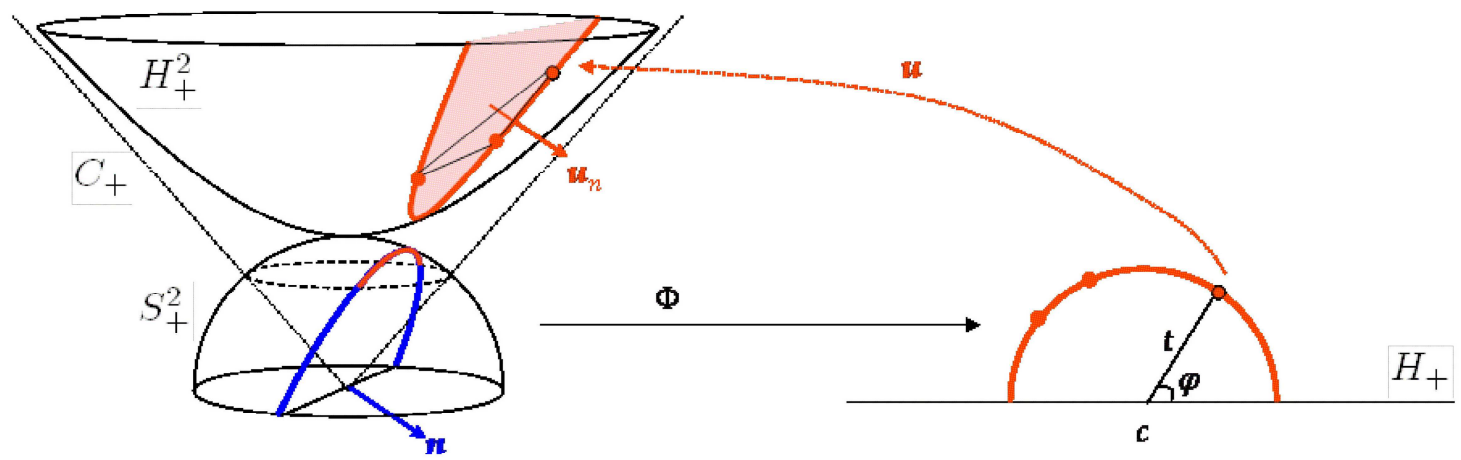

Figure 7. The projection of the arcs of the semi-great circles on $S_{+}^{2} \cup C_{+}$onto $H_{+}$by $\Phi$, and vice versa.

where $\Phi^{*}$ is the pull-back of $\Phi$ defined by $\Phi^{*} f\left(y_{2}, y_{3}\right)=f(\Phi(\boldsymbol{x}))$, with $\Phi(\boldsymbol{x})=\boldsymbol{y}=$ $\left(1, y_{2}, y_{3}\right),\|\boldsymbol{x}\|_{H}=\sqrt{x_{1}^{2}+x_{2}^{2}-x_{3}^{2}}$ is the hyperbolic metric, $d s_{H_{+}}^{2}$ and $d s_{C_{+} \cap S_{+}^{2}}^{2}$ are the associated Riemannian metrics on $H_{+}$and $C_{+} \cap S_{+}^{2}$ restricted to the great circle $Y$.

The left-hand side of (14) defines the hyperbolic x-ray transform $f$, while the right-hand side is the weighted Funk transform of $f(\Phi(\boldsymbol{x}))$.

Proof. Using the parameterization $\boldsymbol{n}=[-\sin \phi \sin \theta, \cos \phi \sin \theta, \cos \theta]^{T}, \boldsymbol{x}=$ $\left[x_{1}, x_{2}, x_{3}\right]^{T}=[\cos \phi \cos \alpha-\sin \phi \cos \alpha, \sin \phi \cos \alpha+\cos \phi \cos \theta \sin \alpha,-\sin \theta \sin \alpha]^{T}$, the associated Riemannian metrics on $H_{+}$and $C_{+} \cap S_{+}^{2}$ restricted to the great circle $Y$ are given by

$$
\begin{aligned}
& d s_{C_{+} \cap S_{+}^{2}}^{2}=d x_{1}^{2}+d x_{2}^{2}+d x_{3}^{2}=d \alpha^{2} \\
& d s_{H_{+}}^{2}=\frac{d y_{2}^{2}+d y_{3}^{2}}{y_{3}^{2}} .
\end{aligned}
$$

Consequently,

$$
\begin{gathered}
\Phi^{*} d s_{H_{+}}^{2}=\frac{d y_{2}^{2}+d y_{3}^{2}}{y_{3}^{2}}=\frac{\sum_{k=1}^{2}\left(\sum_{l=1}^{3} \frac{\partial y_{k}}{\partial x_{l}} \frac{\partial x_{l}}{\partial \alpha} d \alpha\right)^{2}}{\left(\Phi(\boldsymbol{x}) \cdot \boldsymbol{e}_{3}\right)^{2}}=\frac{n_{1}^{2}+n_{2}^{2}-n_{3}^{2}}{\left(x_{1}^{2}+x_{2}^{2}-x_{3}^{2}\right)^{2}} d \alpha^{2} \\
=\frac{\|\boldsymbol{n}\|_{H}^{2}}{\|\boldsymbol{x}\|_{H}^{4}} d s_{C_{+} \cap S_{+}^{2}}^{2} .
\end{gathered}
$$

Note that, by (16), the hyperbolic x-ray transform is a weighted circular averages transform, where the weight is given by $1 / y_{3}$. Thus we have:

Theorem 4.2 The circular averages and Funk transforms are related by:

$$
\mathcal{R}_{C}[f](c, t)=\frac{\|\boldsymbol{n}(c, t)\|_{H}}{t} \mathcal{M}\left[\frac{f(\Phi(\boldsymbol{x}))\left[\Phi(\boldsymbol{x}) \cdot \boldsymbol{e}_{\mathbf{3}}\right]}{\|\boldsymbol{x}\|_{H}^{2}}\right](\boldsymbol{n}(c, t))
$$


where

with

$$
\boldsymbol{n}(c, t)=\frac{\boldsymbol{u}_{\boldsymbol{n}}(c, t)}{\left|\boldsymbol{u}_{\boldsymbol{n}}(c, t)\right|}
$$

$$
\boldsymbol{u}_{\boldsymbol{n}}(c, t)=\frac{3}{8}\left(\frac{4+t^{2}-c^{2}}{4 t}, \frac{c}{t}, \frac{-4+t^{2}-c^{2}}{4 t}\right) .
$$

and $\Phi(\boldsymbol{x})=(c+t \cos \theta, t \sin \theta)$, for $\boldsymbol{x} \in C_{+} \cap S_{+}^{2}$.

Proof. In order to map the circular averages transform to the Funk transform, one needs to determine which semi-circle on the half-plane is mapped to which semi-great circle on the upper-hemisphere, i.e., given $(c, t)$, one needs to determine the normal $\boldsymbol{n}(c, t)$ of the plane that encloses the corresponding semi-great circle. The derivation of $\boldsymbol{n}(c, t)$ is given in Appendix A.

Then, for $\left(y_{2}, y_{3}\right)=(c+t \cos \theta, t \sin \theta)$, substituting (15) in (14) we have

$$
t \mathcal{R}_{C}[g](c, t)=\|\boldsymbol{n}(c, t)\|_{H} \mathcal{M} G(\boldsymbol{n}(c, t))
$$

where $g\left(y_{2}, y_{3}\right)=y_{3}^{-1} f\left(y_{2}, y_{3}\right)$, for $\left(y_{2}, y_{3}\right)=(c+t \cos \theta, t \sin \theta)$ and $G(\boldsymbol{x})=F(\boldsymbol{x}) /\|\boldsymbol{x}\|_{H}^{2}$, where $F(\boldsymbol{x})=f(\Phi(\boldsymbol{x}))$, for $\boldsymbol{x} \in C_{+} \cap S_{+}^{2}$. Replacing $g\left(y_{2}, y_{3}\right)$ with $y_{3} g\left(y_{2}, y_{3}\right)$ in (21) completes the proof.

\subsection{Inversion of Circular Averages Transform}

The inversion of the circular averages follows from (18) and is given in the following corollary:

Corollary 4.3 The circular averages transform can be inverted by the following formula:

$$
f\left(y_{2}, y_{3}\right)=\frac{\left\|\Phi^{-1}\left(y_{2}, y_{3}\right)\right\|_{H}^{2}}{y_{3}} \mathcal{M}^{-1}\left[\frac{t \mathcal{R}_{c}[f](c, t)}{\|\boldsymbol{n}(c, t)\|_{H}}\right]\left(\Phi^{-1}\left(y_{2}, y_{3}\right)\right)
$$

where $\Phi(\boldsymbol{x})=\left(y_{2}, y_{3}\right)$.

The inversion formula above can be implemented in six steps as:

Step 1. Compute $F_{1}(c, t)=\frac{t \mathcal{R}_{C}[f](c, t)}{\|\boldsymbol{n}(c, t)\|_{H}}$ by multiplying $\mathcal{R}_{C}[f](c, t)$ with $\frac{t}{\|\boldsymbol{n}(c, t)\|_{H}}$.

Step 2. Compute $F_{2}(\boldsymbol{n}(c, t))=\frac{t \mathcal{R}_{c}[f](c, t)}{\|\boldsymbol{n}(c, t)\|_{H}}$ by projecting $t /\|\boldsymbol{n}(c, t)\|_{H} \mathcal{R}_{C}[f](c, t)$ onto the unit sphere using $\Phi^{-1}$.

Step 3. Compute $F_{3}(\boldsymbol{x})=\mathcal{M}^{-1}\left[F_{2}\right](\boldsymbol{x})$ by taking the inverse Funk transform of $F_{2}(\boldsymbol{n})$.

Step 4. Compute $F_{4}(\boldsymbol{x})$ by multiplying $F_{3}(\boldsymbol{x})$ with $\|\boldsymbol{x}\|_{H}^{2}$.

Step 5. Compute $F_{5}(\Phi(\boldsymbol{x}))=F_{4}(\boldsymbol{x})$.

Step 6. Compute $f(\Phi(\boldsymbol{x}))$ by multiplying $F_{5}(\Phi(\boldsymbol{x}))$ with $1 / y_{3}$.

Figure 8 summarizes the above steps graphically. Figure 9 illustrates the steps of the reconstruction algorithm for a circular disk phantom. For the parameters of the disk phantom, see Table 2, Phantom 1.

Note that for truncated circular averages data, Step 2 results in truncated limited data on the sphere. In order to reduce the artifact caused by this truncation, we performed linear interpolation in spherical coordinates to fill the truncated data on the sphere. 


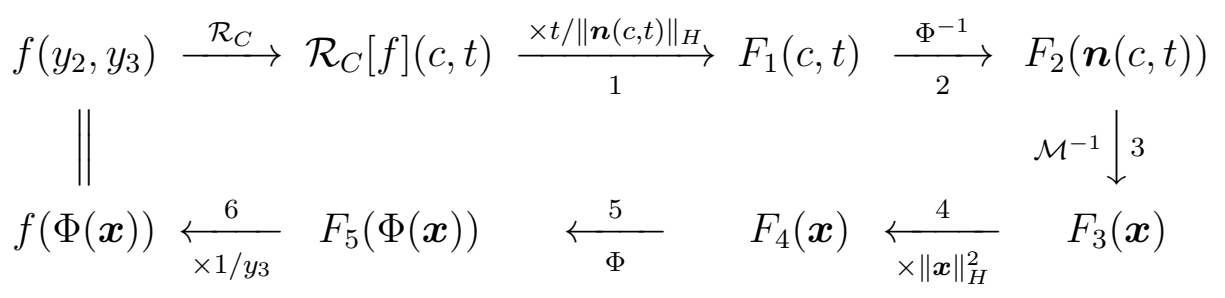

Figure 8. The steps of the algorithm for the inversion of the circular averages transform using the Funk transform.

\section{Numerical Simulations}

We used numerical phantoms to demonstrate the performance of the new inversion method.
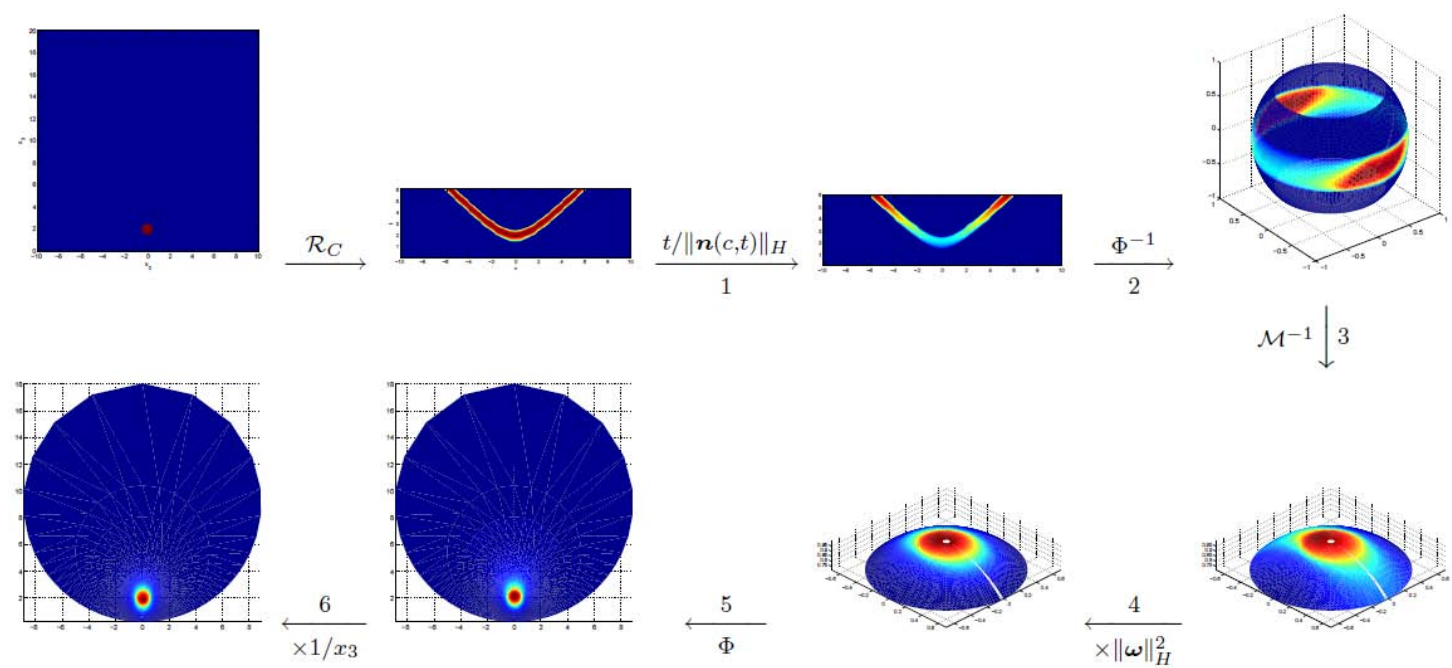

Figure 9. The steps of the inversion of the circular averages transform using the Funk transform for the disk phantom 1. For the parameters of the disk phantom see Table 2.

We computed the circular averages transform of the disk phantoms analytically. Let

$$
f\left(x_{1}, x_{2}\right)=\left\{\begin{array}{cc}
1 & \text { for }\left(x_{1}-c_{0}\right)^{2}+\left(x_{2}-y_{0}\right)^{2} \leq r^{2} \\
0 & \text { otherwise }
\end{array},\right.
$$

be a disk centered at $\left(c_{0}, y_{0}\right), y_{0}>0$, with radius $r<y_{0}$. Then

$$
\mathcal{R}_{C} f(c, t)=\left\{\begin{array}{cc}
2 \varphi t, & 0<t-r<\sqrt{\left(c-c_{0}\right)^{2}+y_{0}^{2}}<t+r \\
0, & \text { otherwise }
\end{array}\right.
$$

where $\varphi$ is given by the law of cosines (see Figure 10) by

$$
\left[\left(c-c_{0}\right)^{2}+y_{0}^{2}\right]+t^{2}-2 t \sqrt{\left(c-c_{0}\right)^{2}+y_{0}^{2}} \cos \varphi=r^{2} .
$$

We reconstructed disk phantoms with unit amplitude and varying radii as shown in Figure 12. The center parameters and radii of the disks are given in Table 2. We formed the circular 


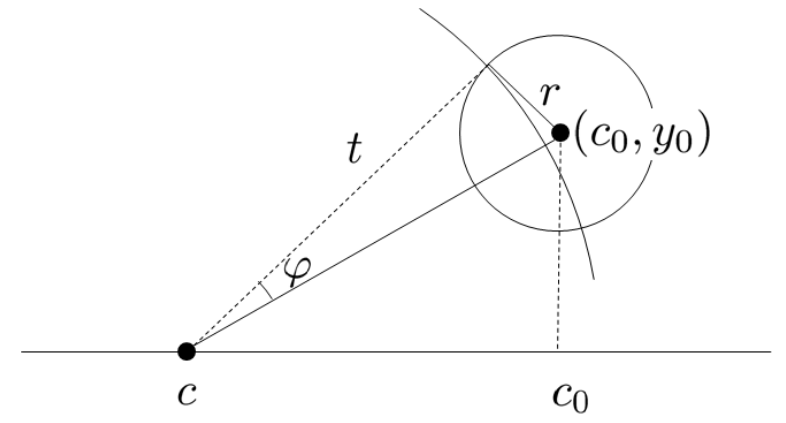

Figure 10. $\varphi$ is given by the law of cosines as shown in (25).

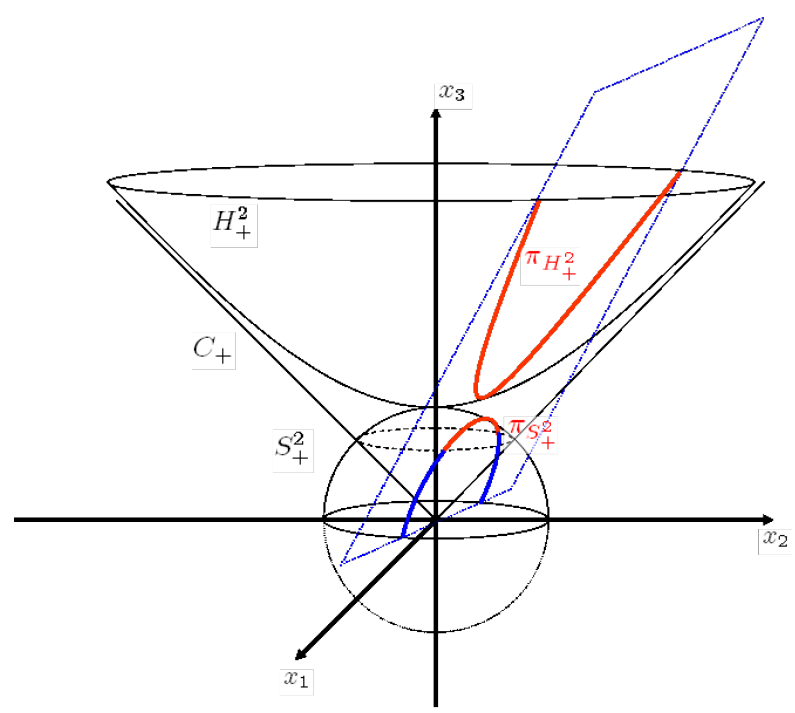

Figure 11. $\pi_{H_{+}^{2}}$ projects arcs of semi-great circles of $S_{+}^{2}$ lying in $C_{+}$onto the hyperbolas given by the intersection of the plane containing the semi-great circle and $H_{+}^{2}$. Conversely, $\pi_{S_{+}^{2}}$ projects hyperbolas obtained by the intersection of $H_{+}^{2}$ and planes passing from the origin onto the arcs of semi-great circles that lie in these planes and $C_{+}$.

averages transform data by uniformly sampling $(c, t)$ on $[-10,10] \times[0,6]$ at $201 \times 119$ points. The scene to be reconstructed is $\left(x_{1}, x_{2}\right) \in[-10,10] \times[0,20]$, which we uniformly discretized into $201 \times 201$ pixels. Phantom 3 is the smallest phantom that we were able to reconstruct whose reconstruction formed the point spread function for our numerical implementation. Figure 13 shows the horizontal cross-section of the reconstructed image for Phantom 3 . The cross section suggests that we can resolve two disks with radii .125 if their edges are .25 apart as demonstrated in Figure 14.

The computational complexity of our inversion method is determined by the computational requirements of the $S O(3)$-Fourier transform implementation, which is needed for the numerical inversion of the Funk transform. In our numerical simulations, we used the $S O(3)$-Fourier transform algorithm presented in [18]. For a function defined on $S^{2}$, which is 


\begin{tabular}{ccc}
\hline Phantom & $\left(c_{0}, y_{0}\right)$ & $r$ \\
\hline 1 & $(0,2)$ & .5 \\
2 & $(0,2)$ & .25 \\
3 & $(0,2)$ & .125 \\
\hline
\end{tabular}

Table 2. The phantom parameters. $\left(c_{0}, y_{0}\right)$ denotes the center of the disks and $r$ denotes the corresponding radius.

\section{Phantom 1}
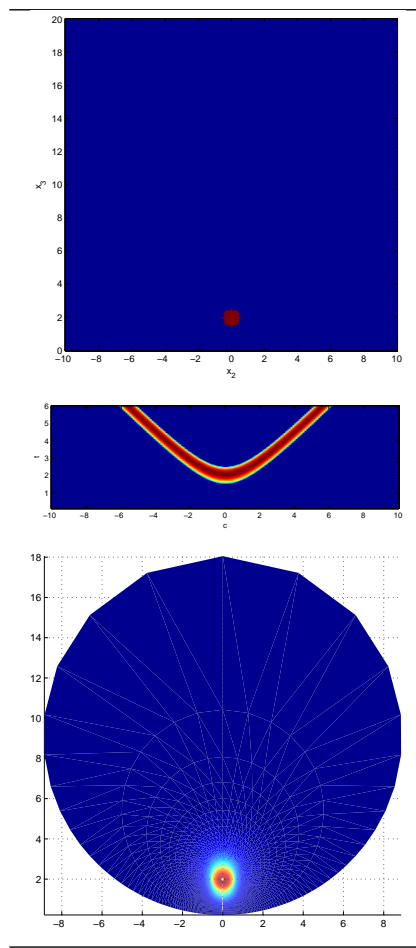

Phantom 2
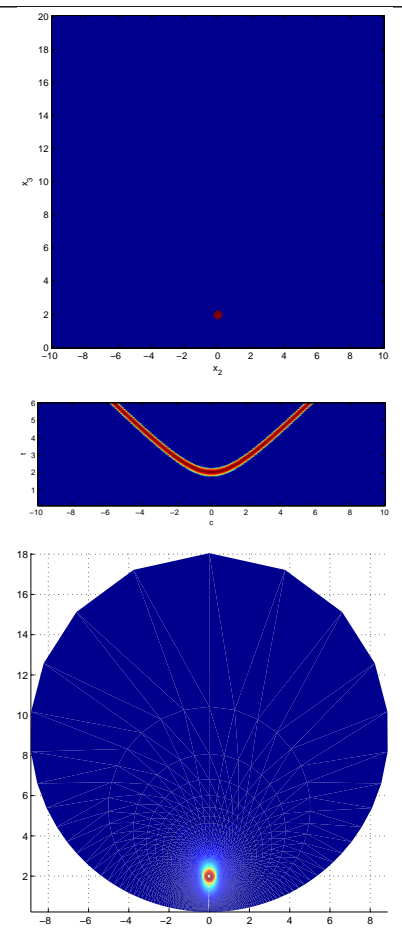

Phantom 3
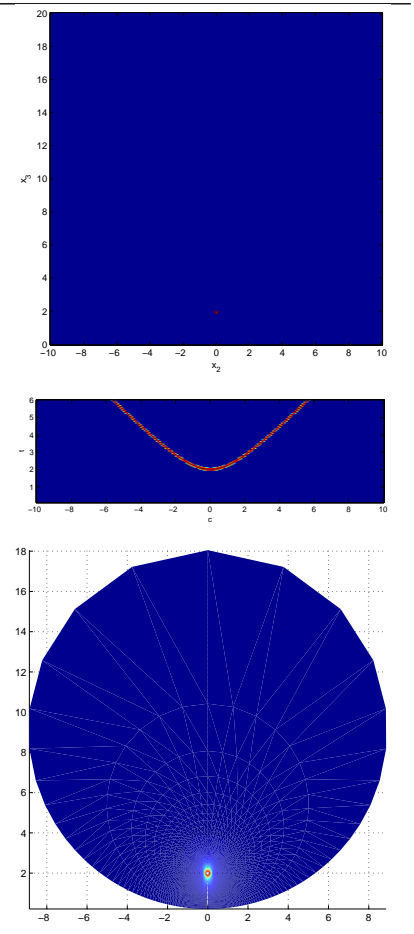

Figure 12. The reconstruction of disk phantoms whose parameters are given in Table 2. (Top) Numerical phantoms, (middle) corresponding circular averages transform data and (bottom) reconstructed images.

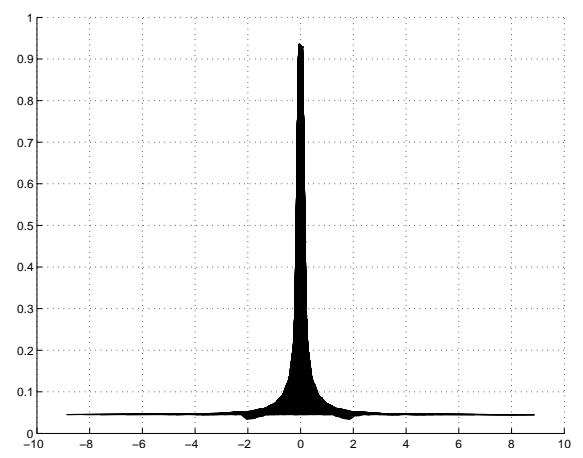

Figure 13. The horizontal cross-section of the point spread function (left) for a disk (right) centered at $\left(c_{0}, y_{0}\right)=(0,2)$ with radius .125 . 


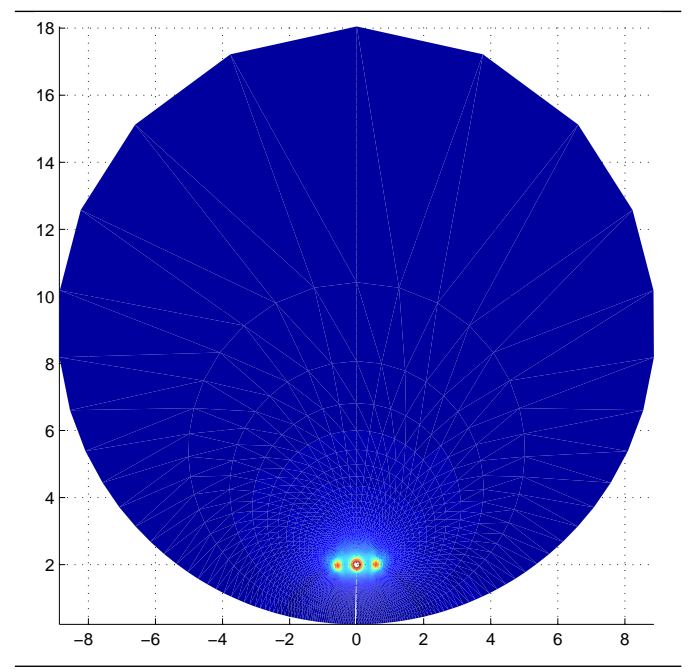

Figure 14. The reconstruction of disks centered at $(-5,2),(0,2)$ and $(-5,2)$ with radius .125 .

uniformly sampled at $2 M \times 2 M$ points in spherical coordinates, the computational complexity of computing its $S O(3)$-Fourier coefficients using the algorithm in [18] is $\mathcal{O}\left(M^{2} \log ^{2} M\right)$. With the recent advancements in the numerical spherical harmonic decomposition methods [21, 22], $S O(3)$-Fourier transform can be implemented with the computational complexity of $\mathcal{O}\left(M^{2} \log M\right)$. Thus, the computational complexity of our inversion method is less than that of the standard filtered backprojection algorithms, which have the computation complexity of $\mathcal{O}\left(M^{3}\right)$, and equal to that of the fast backprojection algorithms, which have the computational complexity of $\mathcal{O}\left(M^{2} \log M\right)[11,30]$.

\section{Conclusion}

We presented a new method for the inversion of the circular averages transform using the Funk transform. In our treatment, we used the relationship between the hyperbolic geometry, circular averages transform and the Funk transform. We viewed the circular averages transform as the hyperbolic x-ray transform on the hyperbolic half-plane and showed that the inversion of the circular averages transform is equivalent to the inversion of the Funk transform for a class of compactly supported functions on the sphere, and developed a new inversion method. We demonstrated the performance of the inversion method in numerical simulations. With the recent advancements in numerical spherical harmonic decomposition, the computational complexity of our inversion method is equal to that of the fast backprojection algorithms.

Our approach can be extended to the inversion of x-ray transform on the hyperbolic disk which has applications in the linearized electrical impedance tomography inverse problem $[31,32,33]$. 


\section{Acknowledgments}

This work is supported by the Air Force Office of Scientific Research (AFOSR) under the agreements FA9550-04-1-0223, FA9550-07-1-0363 and FA9550-09-1-0013.

\section{References}

[1] Andersson L E 1988 On determination of a function from spherical averages SIAM J. Math. Anal. 19 215-232

[2] Nolan C J and Cheney M 2002 Synthetic aperture inversion Inverse Problems 18 221-236

[3] Kruger R A, Reinecke D R and Kruger G A 1999 Thermoacoustic computed tomography Med. Phys. 26 1832-1837

[4] Louis A K and Quinto E T 2000 in D Colton, H W Engl, A K Louis, J R McLaughlin and W Rundell, eds, Surveys on Solution Methods for Inverse Problems (Vienna: Springer-Verlag) pp 147-154

[5] Hellsten H and Andersson L E 1987 An inverse method for the processing of synthetic aperture radar data Inverse Problems 3 111-124

[6] Courant R and Hilbert D 1962 Methods of Mathematical Pyhscis, Vol. II, Partial Differential equations (New York: John Wilter)

[7] Lavrent'ev M, Romanov V and Vasiliev V 1970 Multidimensional Inverse Problems for Differential Eqiuatons vol 167 of Lecture Notes in Mathematics (Berlin: Springer-Verlag)

[8] Norton S 1980 Reconstruction of a reflectivity field from line integrals over circular paths The Journal of the Acoustical Society of America 67(3) 853-863

[9] Palamodov V P 2004 Reconstructive Integral Geometry (Springer Verlag)

[10] Fawcett J 1985 Inversion of $n$-dimensional spherical averages SIAM J. Appl. Math. 45(2) 336-341

[11] Nilsson S 1997 Fast backprojection, technical report lith-isy-r-1865 Tech. rep. Linköping University: Department of Electrical Engineering

[12] Yarman C 2006 Inversion of Radon, exponential Radon, and Funk transforms based on harmonic analysis over groups Ph.D. thesis Rensselaer Polytechnic Institute Troy, NY

[13] Natterer F 1986 The Mathematics of Computerized Tomography (New York, NY: Wiley-Teubner)

[14] Funk P 1913 Über Flächen mit lauter geschlossenen geodätischen Linien (on surfaces with louder closed geodesic lines) Math. Ann. 74 278-300

[15] Driscoll J R and Healy D 1994 Computing Fourier transforms and convolutions on the 2-sphere Advances in Applied Mathematics 15 202-250

[16] Maslen D and Rockmore D 1997 in L Finkelstein and W Kantor, eds, Proceedings of the DIMACS Workshop on Groups and Computation, June 7-10, 1995 pp 183-237

[17] Suda R and Takami M 2001 A fast spherical harmonics transform algorithm Mathematics of computation $71703-715$

[18] DM Healy J, Rockmore D, Kostelec P and Moore S 2003 FFTs for the 2-sphere: Improvements and variations J. Fourier Anal. Appl. 9 341-385

[19] Kostelec P and Rockmore D N 2003 FFTs on the rotation group santa Fe Institute Working Papers, Paper\#03-11-060

[20] D M Healy J, Kostelec P J and Rockmore D 2004 Towards safe and effective high-order Legendre transforms with applications to FFTs for the 2-sphere Adv. Comput. Math. 21 59-105

[21] Rokhlin V and Tygert M 2006 Fast algorithms for spherical harmonic expansions SIAM Journal on Scientific Computing 27 1903-1928

[22] Tygert M 2008 Fast algorithms for spherical harmonic expansions, II Journal of Computational Physics 227 4260-4279

[23] Vilenkin N J 1988 Special functions and the theory of representations (Providence, RI: American Mathematical Society) 
[24] Wigner E 1959 Group theory and its application to the quantum mechanics of atomic spectra (New York: Academic Press)

[25] Varshalovich D, Moskalev A and Khersonskii V 1988 Quantum theory of angular momentum (Singapore: World Scientific)

[26] Ryan P 1986 Euclidean and non-Euclidean geometry : an analytical approach (Cambridge; New York: Cambridge University Press)

[27] Cannon J W, Floyd W J, Kenyon R and Parry W R 1997 Hyperbolic geometry vol 31 of Mathematical Sciences Research Institute Publications (Cambridge, UK: Cambridge Univ. Press) pp 59-115

[28] Anderson J 1999 Hyperbolic geometry (London ; New York: Springer)

[29] Helgason S 2000 Groups and Geometric Analysis: Integral Geometry Invariant Differential Operators, and Spherical Functions (Providence, RI: American Mathematical Society)

[30] Ulander L, Hellsten H and Stenström G 2003 Synthetic-aperture radar processing using fast factorized back-projection IEEE Transactions on Aerospace and electronic systems 39 760-776

[31] Berenstein C A and Casadio Tarabusi E 1993 The inverse conductivity problem and the hyperbolic X-ray transform Tech. rep. The Institute for Systems Research

[32] Berenstein C A and Casadio Tarabusi E 1994 in 75 years of Radon transform (Vienna, 1992), Conf. Proc. Lecture Notes Math. Phys., IV (Cambridge, MA: Internat. Press) pp 39-44

[33] Berenstein C A and Casadio Tarabusi E 1996 Integral geometry in hyperbolic spaces and electrical impedance tomography SIAM J. Appl. Math. 56 755-764

\section{Appendix A. Analytic Derivation of $\boldsymbol{n}(c, t)$}

The circular averages transform inversion formula given in (22) requires computation of the normal vector $\boldsymbol{n}$. $\boldsymbol{n}$ is the normal of the plane that contains the semi-great circle in $S_{+}^{2}$ and the arc which is the inverse image of a semi-great circle in $H_{+}$with respect to $\Phi$ (see Figure 7).

Note that both projections of a semi-great circle in $H_{+}$onto $H_{+}^{2}$ and $S_{+}^{2}$ by $\pi_{\left(H_{+}^{2} \rightarrow S_{+}^{2}\right)}^{-1}$ ○ $\pi_{(-1,0,0)}^{-1}$ and $\pi_{S_{+}^{2}} \circ \pi_{\left(H_{+}^{2} \rightarrow S_{+}^{2}\right)}^{-1} \circ \pi_{(-1,0,0)}^{-1}$, respectively, lie within the same plane. Since three points determine a plane, it is sufficient to determine the projections of three distinct points on the semi-circle in $H_{+}$onto $H_{+}^{2}$ (see Figure 7).

The inverse of $\pi_{(-1,0,0)}$ and $\pi_{\left(H_{+}^{2} \rightarrow S_{+}^{2}\right)}$; and their composition are given by

$$
\begin{aligned}
& \pi_{(-1,0,0)}^{-1}\left(1, x_{2}, x_{3}\right)=\left(\frac{4-\left(x_{2}^{2}+x_{3}^{2}\right)}{4+\left(x_{2}^{2}+x_{3}^{2}\right)}, \frac{4 x_{2}}{4+\left(x_{2}^{2}+x_{3}^{2}\right)}, \frac{4 x_{3}}{4+\left(x_{2}^{2}+x_{3}^{2}\right)}\right) \\
& \pi_{\left(H_{+}^{2} \rightarrow S_{+}^{2}\right)}^{-1}(\boldsymbol{x})=\left(\frac{x_{1}}{x_{3}}, \frac{x_{2}}{x_{3}}, \frac{1}{x_{3}}\right),
\end{aligned}
$$

and

$$
\pi_{\left(H_{+}^{2} \rightarrow S_{+}^{2}\right)}^{-1} \circ \pi_{(-1,0,0)}^{-1}\left(x_{2}, x_{3}\right)=\left(\frac{4-\left(x_{2}^{2}+x_{3}^{2}\right)}{4 x_{3}}, \frac{x_{2}}{x_{3}}, \frac{4+\left(x_{2}^{2}+x_{3}^{2}\right)}{4 x_{3}}\right) .
$$

Using polar representation on $x_{1}=1, x_{3}>0$, a point $p$ on the semi-circle centered at $x_{2}=c$ with radius $r$ can be expressed as $(1, t \cos \varphi+c, t \sin \varphi), t \in \mathbb{R}_{+}$and $\varphi \in(0, \pi)$.

Let $\boldsymbol{u}$ be the projection of $p$ onto $H_{+}^{2}$,

$$
\begin{aligned}
\boldsymbol{u}(c, t, \varphi) & =\pi_{\left(H_{+}^{2} \rightarrow S_{+}^{2}\right)}^{-1} \circ \pi_{(-1,0,0)}^{-1}(1, t \cos \varphi+c, t \sin \varphi) \\
& =\left(\frac{4-\left(t^{2}+c^{2}+2 t c \cos \varphi\right)}{4 t \sin \varphi}, \frac{t \cos \varphi+c}{t \sin \varphi}, \frac{4+t^{2}+c^{2}+2 t c \cos \varphi}{4 t \sin \varphi}\right) .
\end{aligned}
$$


For distinct $\varphi_{0}, \varphi_{1}$ and $\varphi_{2}$, the normal $\boldsymbol{n}$ of the plane passing from $\boldsymbol{u}\left(c, t, \varphi_{0}\right), \boldsymbol{u}\left(c, t, \varphi_{1}\right)$, and $\boldsymbol{u}\left(c, t, \varphi_{2}\right)$ is given by

$$
\boldsymbol{n}(c, t)=\frac{\boldsymbol{u}_{\boldsymbol{n}}(c, t)}{\left|\boldsymbol{u}_{\boldsymbol{n}}(c, t)\right|}
$$

where

$$
\boldsymbol{u}_{\boldsymbol{n}}(c, t)=\left[\boldsymbol{u}\left(c, t, \varphi_{0}\right)-\boldsymbol{u}\left(c, t, \varphi_{1}\right)\right] \times\left[\boldsymbol{u}\left(c, t, \varphi_{0}\right)-\boldsymbol{u}\left(c, t, \varphi_{2}\right)\right] .
$$

Here, $\times$ denotes the vector cross-product. The normal of the plane is independent of the three points chosen on the semi-circle. For computational ease, we choose $\varphi_{0}=\pi / 2$, $\varphi_{1}=\arcsin (0.6)$, and $\varphi_{2}=\arcsin (-0.6)$. Hence

$$
\begin{aligned}
& \sin \varphi_{0}=1, \quad \cos \varphi_{0}=0 \\
& \sin \varphi_{1}=0.8, \cos \varphi_{1}=0.6 \\
& \sin \varphi_{2}=0.8, \cos \varphi_{2}=-0.6
\end{aligned}
$$

which results in

$$
\boldsymbol{u}_{\boldsymbol{n}}(c, t)=\frac{3}{8}\left(\frac{4+t^{2}-c^{2}}{4 t}, \frac{c}{t}, \frac{-4+t^{2}-c^{2}}{4 t}\right) .
$$

